\title{
Supervision und Coaching als Resonanzraum für berührende Transformation
}

\author{
Renate Schwarz
}

Eingegangen: 25. September 2020 / Angenommen: 29. Oktober 2020 / Online publiziert: 28. Januar 2021 (C) Der/die Autor(en) 2021

Zusammenfassung Im Zusammenhang von Supervision und Coaching wird folgender Dreiklang untersucht: das auf einem phänomenologischen Ansatz basierende Prinzip Resonanz und die damit entstehende Intersubjektivität; das Phänomen der Transformation als möglicher Effekt von Resonanz; die Entstehung eines Resonanzraums, eines Ermöglichungsraums für berührende, das heißt sinnerweiternde Transformation im Rahmen von Beratung.

Schlüsselwörter Resonanz · Transformation · Resonanzraum

\section{Supervision and coaching as resonance room for touching transformation}

\begin{abstract}
In the context of supervision and coaching, the following triad is examined: the principle of resonance based on a phenomenological approach and the inter-subjectivity that arises from it; the phenomenon of transformation as a possible effect of resonance; the emergence of a resonance space, an enabling space for touching, i.e. sense-expanding transformation in the context of counselling.
\end{abstract}

Keywords Resonance · Transformation · Resonance Space

Prof. Dr. R. Schwarz ( $\square)$

Hochschule Ravensburg-Weingarten, Postfach 3022, 88216 Weingarten, Deutschland

E-Mail: renate.schwarz@rwu.de 


\section{Einführung}

„Die Dinge sind alle nicht so faßbar und sagbar, als man uns meistens glauben machen möchte; die meisten Ereignisse sind unsagbar, vollziehen sich in einem Raume, den nie ein Wort betreten hat ... “(Rilke 1929, S. 7).

Die Angst, durch intellektuelle Annäherung und Versprachlichung dessen, was mir für Beratung wichtig erscheint, was berührt und transformiert, zu entzaubern, bevor es erlebbar gemacht wurde, prägt den Anfang des Schreibens. Die Kunst, „embodied knowledge“, ,embodied understanding“ und ,lived experience“ zusammenzuführen und die Dynamik und Interdependenz von „Body-mind-world“ für Beratung sichtbar zu machen, ist die Absicht beim Schreiben dieses Artikels.

Folgende Fragen entstehen: Werden Sie den Artikel nach den ersten Zeilen weiter lesen? Wird er, falls Sie ihn lesen, eine Resonanz in Ihnen auslösen, die Sie inspiriert? Falls ja, welche? Werden Sie darüber hinaus in Ihrer alltäglichen Beratungstätigkeit Resonanz bewusster wahrnehmen? Falls ja, wird sich die Qualität Ihrer Arbeit dadurch verändern? Und - werden Sie nach all dem, falls Sie soweit gekommen sind, in Resonanz mit mir treten? Nichts ist unberechenbarer als das, was sich in einem gemeinsam geteilten Raum, auch in einem gemeinsam geteilten Beratungsraum ereignet. Also, lassen wir uns überraschen.

Zur Einführung sei zunächst das Vorgehen erklärt. Basierend auf der von der Phänomenologie geprägten Resonanztheorie von Hartmut Rosa (2017) wird in diesem Artikel folgender Dreiklang untersucht: das Prinzip der Resonanz im Kontext von Beratung und die dadurch entstehende Intersubjektivität und das, was mit Supervision und Coaching meistens anvisiert wird, eine Transformation bzw. Veränderung von Wahrnehmungs- und Denkweisen, Fühlen, Wollen und Zielen oder Handeln und Verhalten in beruflichen Kontexten.

Um in Supervision und Coaching einen Resonanzraum für Veränderung entstehen $\mathrm{zu}$ lassen, ist es entscheidend, dass sich Beratende und $\mathrm{zu}$ Beratende in einer lebendigen und bewegten Art in Verbindung setzen und sich voneinander berühren lassen. Dann entsteht ein vibrierender Raum, ein Zwischenraum, in dem Neues entwickelt werden kann. Dies geschieht, wenn es gelingt, in einer Form der Anstrengungslosigkeit. Um zu dieser Leichtigkeit zu kommen, diesem Flow in einem Beratungsprozess folgen zu können, bedarf es besonderer Wachheit und Bewusstheit von Seiten des oder der Beratenden für eigene Wahrnehmungs-, emotionale und kognitive Prozesse, für Resonanz bei der zu beratenden Person und für den entstehenden Resonanzraum, in dem Transformation ermöglicht wird. Dies zu beschreiben wird im abschließenden Teil versucht.

\section{Resonanz und Leiblichkeit}

Resonanz ohne Leiblichkeit ist nicht denkbar und nicht erlebbar. Resonanz ist zutiefst leiblich. Wenn wir mit anderen Menschen kommunizieren oder interagieren, geschieht dies immer leiblich, meist unbewusst. Wenn wir geschult sind, erleben wir 
diesen Prozess bewusst und können ihn auch für eine gelingende und zielführende Kommunikation nutzen.

„Resonanz meint zuerst und vor allem einen Zustand oder Modus einer dynamischen Beziehung zur Welt, in dem sich Subjekt und Welt (...) wechselseitig berühren und transformieren" (Rosa 2019, S. 17 f.). Wenn Menschen sich von einem Blick oder einer Stimme berührt fühlen, von einem Gedicht, einem Buch, von einer Melodie oder einer Nachrichtensendung, erfolgt eine eigene aktive Antwort. „Diese äußert sich immer auch als eine leibliche Reaktion, die alltagssprachlich etwa in der Entwicklung einer ,Gänsehaut', im ,Sträuben der Nackenhaare ' oder im Schauer, der ,über den Rücken läuft", zum Ausdruck kommt und die sich medizinisch etwa in einer Veränderung der Atemfrequenz oder von Herzschlag und Blutdruck messen lässt“ (ebd., S. 18). Bei Resonanz geht es auch um Spüren - ,,.. dieses Spüren ist die Grundstruktur von Resonanzerfahrung. Das ist dieses In-Beziehung-Treten mit einem Anderen. Und ich glaube, nur wenn man sich den Sinn für dieses Geheimnis noch bewahrt, bleibt man resonanzfähig“ (Rosa und Endres 2016, S. 117). Emotional kann Resonanz erlebt werden als positives Gefühl, als Freude, als das Gefühl, geschützt zu sein, oder als Erleben der Weite im Körper, etwa im Brustbereich, und eines klaren, weiten Blicks und der Perspektive in unterschiedliche Richtungen, oder eben als Wahrnehmung der Enge, des Kleinseins, der Scham, des Beschränktwerdens, der Eingrenzung und des Gefühls, als Person nicht wahrgenommen zu werden. All dies sind genuin leibliche Vorgänge.

Hierzu können wir Bezug nehmen auf Erkenntnisse bedeutender aktueller Forscher wie Thomas Fuchs (2013) mit seinem Werk „Das Gehirn - ein Beziehungsorgan“ und Peter Levine (2010) ,Sprache ohne Worte“ und nachfolgender und vertiefender Werke. „Alles Fühlen, Wahrnehmen, Vorstellen, Denken und Tun vollzieht sich also auf der Basis eines leiblichen Hintergrunds, oder mit anderen Worten: Das Subjekt dieser Tätigkeiten ist immer leiblich“ (Fuchs 2013, S. 97). Der Leib ist in diesem Verständnis ,der Resonanzraum aller Stimmungen und Gefühle, die wir empfinden“ (ebd.). Selbst auf eine Online-Sitzung, in dessen Anwendung die meisten Menschen ja inzwischen geübt sind, reagieren wir leiblich: Manche fühlen sich müde nach einer oder mehreren Sitzungen; andere fühlen sich vielleicht erfrischt, nach einer Zeit der Abstinenz des sozialen Miteinanders das spontane Lächeln oder Winken einer Kollegin wahrgenommen oder die vertraute Stimme eines Kollegen wieder gehört zu haben.

Aber nicht nur das Wahrnehmen und Fühlen vollzieht sich leiblich. Selbst das ,vermeintlich ,reine Denken“ vermag sich nicht vom leiblichen Bewusstsein abzulösen, denn wenn mein Denken sich auch hinsichtlich seiner intentionalen Gehalte in allen Räumen und Zeiten frei bewegen kann, so stellt es als Vollzug doch eine Lebenstätigkeit dar, die an mein leibliches Selbstempfinden und ,Hiersein“ gebunden bleibt - an mein ,Befinden`. Für alle Lebensvollzüge bildet der Leib somit den tragenden Grund" (ebd.).

Andere Autor/innen bedienen sich bei dieser leibbezogenen phänomenologischen Perspektive des Begriffs „Embodiment“ (z. B. Koch 2011; Storch et al. 2017; Tschacher und Storch 2012 u. a.). Auch Begrifflichkeiten wie, felt meaning of lived experience“ oder ,embodied meanings" oder ,feeling knowledge“, „,embodied knowledge“ (Todres 2007) gehen in Forschung und Psychologie von einem phänomenologischen 
Primat aus. So untersucht Levine (2010) die Rolle des somatic experiencing, wenn es um die Bearbeitung von Traumata geht. Seine Untersuchungen und Forschungen lassen ihn vom „dreieinigen Gehirn“ (2010, S. 315) sprechen, von der Interdependenz des Neokortex, der der Primatenebene entspricht, dem Limbischen System, das der der Säugetierebene entspricht, und dem Hirnstamm, dem ältesten Teil des Gehirn, der der Ebene unserer sehr frühen Vorfahren, den Reptilien entspricht. Mit seinen Erkenntnissen kommt er zu der Sichtweise, dass das Gehirn die Verfeinerung der evolutionsgeschichtlich ältesten Bedürfnisse der Organfunktionen enthält. Die Konsequenz daraus ist, dass wir ,aus dem Bauch heraus denken und fühlen“ (ebd., S. 310). Seine Schlussfolgerung für die Arbeit mit Menschen, die ein Trauma oder komplexe Traumata erfahren haben, ist folgende: Um heilsam und unterstützend wirken zu können, müssen Worte immer in einem physischem Kontext stehen (Levine 2010, S. 322). Der Ausgangspunkt für Heilung bzw. Veränderung geht nur über Miteinbeziehen des Körpers und einer embodied stance (De Jaeger et al. 2017, S. 500), einer Haltung, die sowohl die körperlich-leiblichen Prozesse des Beraters oder Therapeuten als auch des zu Unterstützenden wahrnimmt.

Lassen Sie uns nach diesen Ausführungen zu phänomenologisch inspirierten Aspekten zur Leiblichkeit zurückkehren - zum Begriff der Resonanz, wie er von Hartmut Rosa vorgeschlagen wird. Resonanz bezeichnet einen ,,spezifischen Modus der Weltbeziehung“ (Rosa 2019, S. 16) oder „einen Modus des In-der-Welt-Seins, das heißt eine spezifische Art und Weise des In-Beziehung-Tretens zwischen Subjekt und Welt" (2017, S. 285). Ein Mensch wird durch die Welt, das kann ein anderer Mensch, etwa ein Coach, ein Raum, ein Verbot sein, ,,so berührt oder bewegt, dass ein intrinsisches Interesse an dem begegnenden Weltausschnitt entwickelt" wird und die Person sich ,gleichsam angesprochen fühlt. (...) Auf der anderen Seite lässt sich von Resonanz erst dann sprechen, wenn auf diese Berührung (oder Anrufung) eine eigene, aktive Antwort erfolgt" (Rosa 2019, S. 18). Weiter führt Rosa aus, dass der Mensch angewiesen ist auf Resonanz, dass sie für die menschliche Psyche, die Sozietät sowie für ihre Leiblichkeit konstitutiv wird.

Für die weiteren Ausführungen, die ja die Bedeutung von Resonanz im Kontext Beratung in den Blick nehmen sollen, ist die Resonanzbeziehung von besonderem Interesse. Von einer ,genuinen Resonanzbeziehung“ (ebd.) wird erst dann gesprochen, wenn die Antwort auf etwas Berührendes eigene Selbstwirksamkeit enthält, also wenn das, was ich höre, rieche, sehe, spüre oder auf eine andere Art erlebe, etwas in mir auslöst, ins Schwingen bringt, wenn ein Impuls entsteht, der etwas in Bewegung setzt. Das kann ein Lächeln, der Austausch eines Blicks oder ein Dialog, ein YouTube-Video oder eine von außen auferlegte Begrenzung unseres Freiheitsempfindens oder eine politische Nachricht sein. Etwas in uns wird zum Schwingen gebracht, etwas klingt an, der Ton schwingt weiter und bewirkt eine Art von Veränderung, mag sie nach außen hin noch so unbedeutend erscheinen. Auch dies vollzieht sich leiblich. Aus einer leiblichen Perspektive ist nicht zu unterscheiden, ob ein Gedanke, ein Gefühl, eine Wahrnehmung oder eine Erkenntnis Auslöser dieser Veränderung war. Es entsteht ein ,vibrierender Resonanzdraht zwischen Selbst und Welt" (ebd., S. 17).

Die Einzelnen lassen sich bewegen und berühren und werden gleichzeitig bewegt und berührt. Intersubjektives Agieren wird möglich, und eine intersubjektive Bezie- 
hung entsteht. ,Der Andere erscheint mir primär als lebendig-leibliche Einheit, d.h. er ist selbst in seinem Körper präsent, ,leibhaftig ' anwesend. Sein Körper ist für mich nicht bloß ein anatomischer Träger einer ,Innerlichkeit', die mir unzugänglich bleibt und auf die ich nur aufgrund von geeigneten Vorerfahrungen oder Kenntnissen schließen kann. Die Verkörperung bildet vielmehr die Basis der Intersubjektivität, insofern wir dem Anderen in leiblichen Interaktionen nicht abstrakte innere Zustände zuschreiben, sondern seine Mimik, seine Gestik und sein Verhalten im Situationskontext unmittelbar als Ausdruck seiner Empfindungen und Gefühle erfahren. Die primäre Wahrnehmung Anderer beruht also (...) auf der leiblichen Kommunikation und wechselseitigen Empathie verkörperter Subjekte“ (Fuchs 2013, S. 102).

Diese Intersubjektivität oder zwischenleibliche Resonanz (Fuchs und Koch 2014; vgl. Schwarz 2019) ist möglich, wenn sich zwei berühren lassen. „Unser Leib wird affiziert von dem Gefühlsausdruck eines anderen Menschen, und wir erfahren die Kinetik und Intensität seiner Emotionen an eigenen leiblichen Bewegungsimpulsen und Empfindungen.“ Diese sind zum einen geprägt von ,eigenleiblichen Resonanzen“ und den „zwischenleiblichen Resonanzen“ (Fuchs 2014, S. 17). So entsteht ein zirkulärer Prozess zwischen eigenleiblichen und zwischenleiblichen Resonanzen, der mit einem kreativen Tanz zu vergleichen ist. Rosa bringt dies folgendermaßen zum Ausdruck: „Resonanz (als Zurück-Tönen) bezeichnet das In-Beziehung-Treten zweier Seiten oder Entitäten, die je mit eigener Frequenz schwingen, oder bildlich gesprochen, mit je eigener Stimme sprechen. Für das Subjekt bedeutet dies, einem genuin Anderen als Anderem zu begegnen“" (Rosa 2019, S. 21).

Resonanz im Sinne von Hin- und Herschwingen, sodass etwas Berührendes entstehen und zu Veränderung anregen kann, bedeutet indessen nicht, dass immer Harmonie oder Konsonanz besteht. ,In einer Atmosphäre der (völligen) Harmonie oder Konsonanz finden weder Berührung noch eine selbstwirksame Antwort und erst recht keine Transformation statt. (...) Resonanz bezeichnet damit ein Geschehen, welches sich zwischen den Polen radikaler Dissonanz und reiner Konsonanz ereignet; es setzt Differenz notwendig und unaufhebbar voraus, erlaubt aber Hoffnung auf, und impliziert Möglichkeiten von, , anverwandelnder ' Transformation, die eben nicht einseitige Aneignung, Assimilation oder Nostrifizierung meint, sondern um den Preis des Eigenen zu haben ist“ (ebd.). Offensichtlich aber ist, ,dass uns die Gegenstände als Begegnende durch die Resonanzerfahrung verändern“ (ebd., S. 19). Hier kann dann von Transformation gesprochen werden.

\section{Transformation als Effekt einer Resonanzbeziehung}

Eine resonante Beziehung ermöglicht also per se eine zweiseitige Veränderung, wenn eine berührende Begegnung stattfindet. „Resonanzbeziehungen sind mithin dadurch gekennzeichnet, dass sich mit und in ihnen Subjekt und begegnende Welt verändern“ (Rosa 2019, S. 19). Hierfür verwendet Rosa den Begriff der „Anverwandlung“ im Gegensatz zur „Aneignung“ von Welt. Wenn uns etwas durch ein Anderes oder eine Andere auf eine Art berührt, affiziert und Emotion als eine Antwort durch die Verbindung entsteht, haben wir es mit Anverwandlung zu tun. Entsteht eine Reaktion, die nicht berührt, handelt es sich um Aneignung, um eine Beziehung der 
Beziehungslosigkeit, die keine Veränderung des Denkens, Handelns oder Wollens hinterlässt. So kann es vorkommen, dass wir ein Buch lesen, das in uns nichts zum Schwingen bringt, oder einen Vortrag hören, der nicht nachklingen mag. Als Supervisoren und Coaches zucken wir zusammen, wenn am Ende einer Sitzung eine Teilnehmerin in einer Feedbackrunde mit der Aussage „Eigentlich ist es wie am Anfang der Sitzung“ den Supervisionsprozess resümiert. Eine Resonanzbeziehung konnte nicht entstehen, und es konnte nichts ins Schwingen gebracht werden, was eine Veränderung z. B. einer prekären Situation hätte herbeiführen können.

Folgendes Beispiel aus meiner supervisorischen Praxis, einer ersten Supervisionssitzung mit einem Team, die als Probesupervision kontraktiert war, kann dies deutlich werden lassen.

Nach einen Gespräch mit dem Sprecher einer Gruppe von Teamleiter/innen einer Einrichtung war ich zum vereinbarten Termin vor Ort. Vereinbart war ein gegenseitiges Kennenlernen und Abklären des Auftrags für Leitungssupervision. Meine erste Erfahrung war, dass ich den genauen Ort der Sitzung nicht finden konnte. Der Teamleiter hatte mir den genauen Ort auf Nachfrage nicht genannt und meinte, es würde sich auf dem Gelände schon jemand finden, der mich informieren würde. Da dem nicht so war, verzögerte sich der Zeitpunkt des Beginns der Supervision. Das Team hatte zuvor eine Besprechung und war noch nicht auf unsere Sitzung eingestellt. Der Raum musste neu bestuhlt werden, und mir fiel auf, dass er für die Größe der Gruppe eigentlich zu klein war. Ein Gefühl des Unbehagens machte sich in mir breit, wurde aber durch die Neugierde auf die Teammitglieder, ihre Arbeit und ihre Anliegen für Supervision nicht wirklich ernst genommen.

Das Gefühl der Enge in meinem Brustkorb wurde immer deutlicher, als das Team ein aus seiner Sicht gravierendes Problem mit der Leitung thematisierte, das für mehrere Teammitglieder emotional unlösbar schien. Mein Bemühen, zu verstehen und einen Schritt in Richtung Erleichterung oder Lösung zu entwickeln, erwies sich als unmöglich. Es entstand kein wechselseitiges resonantes Schwingen zwischen Teammitgliedern und mir. Meine Versuche zu intervenieren ließen bei den Teammitgliedern keine Seite in Bewegung bringen, die es in Richtung Transformation, auch nur im Sinne einer gedanklich-sprachlichen Veränderung, hätte bewegen können. Das innere Bild, einer Wand gegenüberzustehen, von der keine Schwingung bei mir ankommen konnte, machte sich in mir breit. Es schien mir und dem Team nicht zu gelingen, eine Resonanzbeziehung mit transformatorischen Effekten entstehen zu lassen. Das bestätigte für mich dann der abschließende Satz einer Teilnehmerin ,Eigentlich ist es wie am Anfang der Sitzung“. Etwas irritiert und ratlos ging in von dannen. Das war ich nicht gewohnt, dass in einer ersten, sondierenden Sitzung eine resonierende Beziehung nicht entstehen konnte. Einige Tage später erhielt ich vom Teamsprecher eine E-Mail mit dem Inhalt, dass das Team sich um eine andere Person für die Supervision umschauen wolle. Das klang als stimmig in mir nach.

Nicht allen Teams, Supervisanden oder Coachees ist es zu jedem Zeitpunkt möglich, in einen „Resonanzmodus“ zu gelangen, wie diese Erfahrung zeigt. Darüber 
hinaus gibt es ein „Recht auf Resonanzverweigerung“ (Rosa 2019, S. 28). Nicht in jedem kollegialen Rahmen und zu jedem Zeitpunkt ist Resonanz, die Differenziertheit in der Wahrnehmung und Sensibilität erfordert, möglich. Zeitnot, Angst, die Gesamtheit der Arbeitsverhältnisse, ein machtvoller Virus oder andere problematische Situationen können Resonanz verunmöglichen. „Transformative Effekte einer Resonanzbeziehung“ oder einer potenziellen Resonanzbeziehung entziehen sich „stets unvermeidlich der Kontrolle und Planung des Subjektes“. „In welcher Weise und in welcher Tiefe wir uns verändern, wenn wir uns auf einen Menschen, eine Lebensform, eine Idee, ein Buch, eine Landschaft wirklich einlassen, lässt sich nicht, oder jedenfalls nicht sicher, vorhersagen." Unverfügbarkeit wird so zu einem Bestimmungsmerkmal von Resonanz. „So gibt es auch keine Möglichkeit, Resonanz systematisch bzw. instrumentell zu erzwingen“ (Rosa 2019, S. 19).

Für Supervision und Coaching heißt das, dass Resonanz gelingen kann und in den überwiegenden Fällen auch gelingt, aber sie kann eben auch nicht gelingen. Man kann weder um Resonanz kämpfen noch den Beziehungsmodus systematisch bzw. instrumentell herbeiführen. ,Sobald wir in eine Kampfbeziehung treten, sind wir zu einer resonanzdämpfenden Schließung gezwungen. Zur Unverfügbarkeit von Resonanz zählt als weiteres Merkmal schließlich auch, dass sie sich nicht akkumulieren, nicht speichern und nicht instrumentalisieren lässt“" (ebd., S. 20).

\section{Resonanzraum für berührende Transformation}

Wie nun entsteht ein Raum, in dem wir uns durch resonante Erfahrungen verändern können, in dem berührende Transformationen möglich sind? Wie entsteht ein Raum z. B. zwischen Lehrenden und Lernenden, etwa in der Schule oder in Vorlesungsräumen, in dem Lernen im Sinne von Aneignung von etwas Neuem und Auseinandersetzung mit Fremdem möglich wird? Ein Raum, in dem wir unser Wahrnehmen, Denken, Fühlen, vielleicht auch unsere Ziele so verändern können, dass sie uns auf eine sinnvolle und berührende Art und Weise in Bewegung bringen? Das ist ja das, was in der Regel mit Supervision und Coaching anvisiert wird.

Es ist ein sozialer Raum, ein Zwischenraum, in dem subtil intersubjektiv oft auch ohne Sprache kommuniziert wird. Man könnte diesen Raum auch mit einem Tanzraum vergleichen, in dem ein Hin- und Herschwingen der sich Bewegenden möglich ist. Je nachdem wie dieser Zwischenraum zwischen zwei oder mehreren Menschen sich gestaltet, ist freie und kreative Bewegung möglich.

Das heißt für Supervision und Coaching, wenn in diesem „Zwischenraum“ ein Dialog zwischen Beratender und zu Beratenden möglich wird, sodass ,ein wechselseitig (affiziertes) ,Hören ' und (selbstwirksames) ,Antworten ' entsteht, werden sich beide Seiten modifizieren, ohne ihre eigene Stimme zu verlieren“ (Rosa 2019, S. 23). Interessant aus dieser intersubjektiven Perspektive ist, dass sich nicht nur die Person, die Beratung in Anspruch nimmt, transformiert, modifiziert oder eben einen gewünschten Aspekt verändert, sondern dass sich dieser Prozess, wenn auch vielleicht in abgeschwächter und nicht intendierter Form, auch bei der Beraterin ereignet. 
Supervisorin/Coach

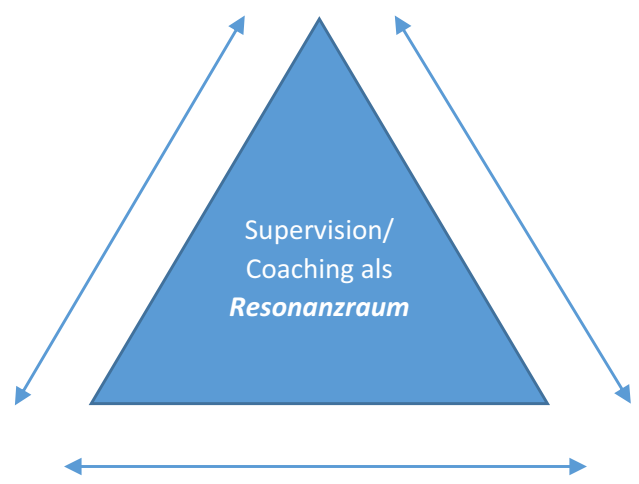

Supervisand/Coachee, Team

Inhalt der Supervision/des Coachings Auftrag, Themen, Anliegen, Ziele etc.

Abb. 1 Das Resonanzdreieck

Es wird ein Raum sein, in dem Kraft zu erfahren ist, ein Raum, in dem es, wenn es eng geworden ist, wieder weiter wird, in dem neue Innenräume entstehen, ungewohnte Blickwinkel und Perspektiven, in dem sich eine oder mehrere Türen für Lösungen öffnen. Es wird ein Raum sein, in dem umhergegangen wird, in dem wahrgenommen, gefühlt, getrauert, geträumt wird, in dem Pläne für weiteres Vorgehen und neue Wegrichtungen und Ziele angedacht werden. Es kann auch ein Raum sein, in dem eine wegweisende berufliche Entscheidung getroffen wird. All diese großen und winzigen Bewegungsimpulse werden erlebt und sind berührende Transformationen. Wenn Supervision und Coaching ein Resonanzraum zwischen Beraterin und zu Beratenden wird, ,dann zieht mich etwas nahezu dahin“, dann ist es ein „Raum mit Anziehungskraft“ (Rosa und Endres 2016, S. 36). Das trifft im Idealfall für beide Seiten zu.

Für den schulischen Kontext schlägt Rosa ein „Resonanzdreieck“ für eine gelungene Stunde vor (ebd., S. 46). Dieses Modell scheint mir auch auf supervisorische Prozesse übertragbar zu sein und wird hier dargestellt (Abb. 1).

Die Supervisorin oder der Coach, Supervisand, die Coachees oder ein Supervisionsteam und der Inhalt, das Thema oder Auftrag bilden die jeweilige Achse des Dreiecks. Die Supervisorin tritt in eine resonante Beziehung mit der Supervisandin, ist neugierig, begeistert und lässt sich von dem, was sie wahrnimmt, hört, sieht, riecht, was in ihr resoniert, berühren. Die Supervisandin oder der Coachee ist von einem Anliegen, einem Problem oder einer Irritation berührt, fühlt sich angenommen und aufgehoben und ist zugleich offen für Veränderung. Das Thema bzw. der Inhalt der Beratungssituation erscheint sowohl der Beratenden als auch der zu Beratenden als bedeutungsvoll und herausfordernd. Der Raum zwischen diesen drei Feldern lässt Möglichkeiten der Transformation entstehen. Dieser Coachingraum ist ein geschützter Raum, von dem verbindlich zugesagt nichts nach außen dringen soll. Es ist ein ,abgeschirmter Interaktionsraum“ (Erpenbeck 2020, S. 96). Das macht ihn so kostbar. Und - es können sich durch „Methodik“ und „Instrumente“ 
„organisch und geschmeidig ein paar neue Tanzfiguren in den Rhythmus der Bewegung einfügen“ (ebd.). Methoden und Instrumente sind im Sinne der Bedeutung des beschriebenen intersubjektiven Geschehens indessen zweitrangig. Sie entfalten aus dieser Prämisse heraus betrachtet ihre Wirkung eh nur dann, wenn ein schwingender, resonanter Raum bereits vorhanden ist. Anders ausgedrückt, wenn das „Unbewusste der beiden Rollenträger“ (ebd., S. 16) in Resonanz getreten ist. Zuweilen kann ich als Supervisorin die inneren Zustände meines Supervisanden an dessen körperlichen Reaktionen ableiten, ,der wortlosen Sprache seiner Aktivität, Inaktivität oder körperlichen Spannung“ (Levine 2010, S. 184).

Die Verfügbarkeit dieses resonanten Raumes und die damit verbundene ,subjektiv erlebte Resonanzerfahrung sind stets temporäre, unverfügbare Ereignisse; sie können nicht auf Dauer gestellt werden“ (Rosa 2019, S. 24). Als Coaches oder Supervisoren haben wir, wenn wir resonanzsensibel vorgehen, ein genaues Gespür dafür, wann dieser Resonanzraum aktiviert und als Bühne freigegeben ist. Es sind Momente im Setting, die sich leicht anfühlen, in denen ein Flow wahrzunehmen ist, in denen wir uns als Beratende oft innerlich zurücklehnen und beobachtend miterleben, wie unser Gegenüber eine Lösung entwickelt, wie nach einer Zeit des Hin- und Herschwingens eine neue Idee im Raum steht, die motiviert und energetisiert. Was genau innerlich im Coachee während eines solchen Prozesses abläuft, muss in diesem Resonanzraum nicht unbedingt benannt werden, kann aber im Nachhinein auf einer Metaebene reflektiert werden, um das Geschehen für zukünftige Situationen nutzbar zu machen.

Wieder mit einem Beispiel aus meiner supervisorischen Praxis möchte ich das Beschriebene veranschaulichen. Es ist das Beispiel einer gelungenen, berührenden Transformation innerhalb einer Teamsupervision in einer Beratungsstelle:

In der Initialrunde zu Beginn der Teamsupervision beschrieb eine Mitarbeiterin ein als unkonkret erlebtes Irritiert-Sein, das mit unangenehmen Gefühlen verbunden ist. Kurz danach äußerte sie den Wunsch, die Arbeit mit einer Klientin während der vergangenen Woche zu thematisieren. Während einer ersten Schilderung der erlebten Situation mit der Klientin geriet die Kollegin, nennen wir sie Frau W., in heftige körperliche Unruhe. Sie beschrieb ein Gefühl, in die Enge getrieben zu sein. Von der Klientin wurden Erwartungen an sie als Beraterin herangetragen, die weit über ihr eigenes professionelles Verständnis hinausgingen. Zu diesem Zeitpunkt war während unserer Sitzung ein resonanzermöglichender Raum entstanden, die Atmosphäre schien geschützt und vertraut zu sein. Frau W. formulierte nach der Schilderung der belastenden Situation die Frage: Wie kann ich wieder in eine professionelle Rolle und ein gutes NäheDistanz-Verhältnis zu meiner Klientin kommen?

Ich konnte sowohl emotionales, resonantes Hin- und Herschwingen auf das Gesagte von Frau W. als auch meinen eigenen inneren Raum resonierend wahrnehmen. Ich bot Frau W. an, in dem Raum des Hier und Jetzt in einem Simultanspiel ihrer Klientin das zu sagen, was sie ihr am liebsten ganz spontan sagen möchte. Das tat sie dann auch. Nach einigen Wiederholungen mit verändertem Tonus und Lautstärke hatte sie die Lösung für ihr Anliegen gefunden. ,So leicht ist das!" sagte sie mehrmals. Sie war sichtlich erstaunt, wie leicht sich die Lösung anfühlte, und sie erlebte sich wieder in ihrer Kraft. Das war auch spürbar 
und sichtbar für uns andere im Raum und wurde anschließend reflektierend in Worte gefasst.

\section{Anregungen für resonanzsensibles Coaching und Supervision}

Worauf ist zu achten, wenn es darum geht, resonanzsensibel in Supervision und Coaching vorzugehen, sodass ein Resonanzraum entstehen kann, in dem eine berührende Transformation, also Veränderung möglich ist? Ich möchte folgende drei Anregungen vorschlagen.

Zum Ersten geht es darum, dass die Beraterin ihren Fokus auf ihre eigene Wahrnehmung, auf das eigene Erleben lenkt und sich Fragen stellt wie z. B.: Was nehme ich wahr? Was spüre ich? Was sehe ich? Was rieche ich? Was höre ich? Was erfüllt meinen inneren Raum? Was resoniert in mir, was tönt wider? Voraussetzung für eine derart differenzierte Selbstwahrnehmung ist, dass sie eine ,tiefe und stabile Beziehung zu ihrem eigenen Körper hat" (Levine 2010, S. 178). Für die Wahrnehmung des eignen Innenraums und die somatische Resonanz hat Eugen Gendlin (1998) den Begriff felt sense, gefühlte Wahrnehmung oder Bedeutung geprägt. Hier geht es um nuancierte Affekte von gefühlter Bedeutung. Wir haben heute viele Möglichkeiten für somatic experiencing, um unsere somatische Wahrnehmung zu verfeinern. Tai Chi, Gi Gong, Yoga, Aikido, Feldenkrais, Tanzimprovisation und Formen der Meditation wie Zen sind einige davon.

Da Resonanz und Transformation nur möglich sind im gegenseitigen Hin- und Herschwingen von mindestens zwei Seiten, ist die Supervisorin in einer resonanzsensiblen Haltung offen für eigene Veränderungen während und nach einem Beratungssetting. Das klingt zunächst anspruchsvoll, geschieht aber, wenn resonante Kommunikation gelingt, mit Leichtigkeit. Neugieriges, waches, flexibles und situationsbezogenes Vorgehen der Beraterin bestimmen dann den Beratungsprozess.

Zum Zweiten geht es darum, wahrzunehmen, was sich in und bei der anderen Person, den anderen Personen ereignet. Welche somatischen Reaktionen sind wahrzunehmen? Wie verändert sich Mimik und Gestik? Wie Emotionen und Gesichtsausdruck? Wie ist die Körperhaltung, die Sitzhaltung? Wie die Atmung und der Blick? Sind subtile Gesten der Hände und der Arme zu beobachten? Gefragt ist anschließend nicht eine Deutung der somatischen Reaktionen, nicht so etwas wie die Entschlüsselung somatischer Signale. Vielmehr geht es darum, zu beobachten, welche somatischen Reaktionen in der Beraterin ausgelöst werden und welche Hypothesen und Fantasien ,zum inneren Erleben meines Gegenübers“ (Erpenbeck 2020, S. 16) entstehen. Was mag mein Gegenüber verbinden mit den geschilderten Situationen, Worten und somatischen Reaktionen? Das kann im weiteren Verlauf im Sinne systemischen Vorgehens mit Hypothesen nutzbar gemacht werden. Gegebenenfalls können, wenn sich die entwickelten Hypothesen nicht als hilfreich erweisen, diese wieder verworfen werden.

Zum Dritten ist der Coach verantwortlich dafür, einen Resonanzraum entstehen zu lassen. Er ist verantwortlich dafür, dass ein resonanter Prozess entstehen kann, ein nach innen und nach außen hin geschützter Raum. Das beginnt damit, dass eine 
vertraute und sichere Atmosphäre im Einzelcoaching oder in einem Team möglich wird. Er ist verantwortlich dafür, den Raum zu ,halten“. Das kann sich darin ausdrücken, dass auch Leerräume gehalten werden, z.B. wenn es Momente gibt, in denen geschwiegen wird.

Eine Haltung des Neugierigseins begleitet den ganzen Prozess. Da Resonanz systematisch und instrumentell nicht zu erzwingen ist, unterliegt sie der Unverfügbarkeit. Resonanz, berührende Transformation und Leichtigkeit sind nicht in allen Beratungsprozessen zu erfahren. Dies zu akzeptieren, entspricht einer professionellen Haltung des Coachs oder der Supervisorin. Aber wenn resonanzsensibel vorgegangen wird und ein lebendiger Resonanzraum mit Bewegung und Transformation entsteht, ist das sowohl super spannend als auch beglückend - für beide Seiten.

Funding Open Access funding enabled and organized by Projekt DEAL.

Open Access Dieser Artikel wird unter der Creative Commons Namensnennung 4.0 International Lizenz veröffentlicht, welche die Nutzung, Vervielfältigung, Bearbeitung, Verbreitung und Wiedergabe in jeglichem Medium und Format erlaubt, sofern Sie den/die ursprünglichen Autor(en) und die Quelle ordnungsgemäß nennen, einen Link zur Creative Commons Lizenz beifügen und angeben, ob Änderungen vorgenommen wurden.

Die in diesem Artikel enthaltenen Bilder und sonstiges Drittmaterial unterliegen ebenfalls der genannten Creative Commons Lizenz, sofern sich aus der Abbildungslegende nichts anderes ergibt. Sofern das betreffende Material nicht unter der genannten Creative Commons Lizenz steht und die betreffende Handlung nicht nach gesetzlichen Vorschriften erlaubt ist, ist für die oben aufgeführten Weiterverwendungen des Materials die Einwilligung des jeweiligen Rechteinhabers einzuholen.

Weitere Details zur Lizenz entnehmen Sie bitte der Lizenzinformation auf http://creativecommons.org/ licenses/by/4.0/deed.de.

\section{Literatur}

Erpenbeck, M. (2020). Wirksam werden im Kontakt. Die systemische Haltung im Coaching (3. Aufl.). Heidelberg: Auer.

Fuchs, T. (2013). Das Gehirn - ein Beziehungsorgan. Eine phänomenologisch-ökologische Konzeption (4. Aufl.). Stuttgart: Kohlhammer.

Fuchs, T. (2014). Verkörperte Emotionen - wie Gefühl und Leib zusammenhängen. Psychologische Medizin, 25(1), 13-20.

Fuchs, T. \& Koch, S. (2014). Embodied affectivity: on moving and being moved. Frontiers in Psychology, 5(508), 1-11.

Gendlin, E. (1998). Focusing. Selbsthilfe bei der Lösung persönlicher Probleme. Reinbek: Rowohlt.

De Jaeger, H., Pieper, B., Clénin, D. \& Fuchs, T. (2017). Grasping intersubjectivity: an invitation to embody social interaction research. Phenomenology and the cognitive Sciences, 16(3), 491-523.

Koch, S. (2011). Embodiment. Der Einfluss von Eigenbewegung auf Affekt, Einstellung und Kognition. Berlin: Logos.

Levine, P. (2010). Sprache ohne Worte. Wie unser Körper Trauma verarbeitet und uns in die innere Balance zurückführt (9. Aufl.). München: Kösel.

Rilke, R. M. (1929). Briefe an einen jungen Dichter. Insel-Bücherei Nr. 406. Frankfurt/M., Leipzig: Insel. Rosa, H. (2017). Resonanz. Eine Soziologie der Weltbeziehung. (6. Aufl.). Berlin: Suhrkamp.

Rosa, H. (2019). Resonanz als Schlüsselbegriff der Sozialtheorie. In J.-P. Wils (Hrsg.), Resonanz. Im interdisziplinären Gespräch mit Hartmut Rosa (S. 11-30). Baden-Baden: Nomos.

Rosa, H. \& Endres, W. (2016). Resonanzpädagogik. Wenn es im Klassenzimmer knistert. Weinheim, Basel: Beltz. 
Schwarz, R. (2019). Zwischenleibliche Resonanz und Intersubjektivität in Beratung, Supervision und Coaching. In B. Wuttig \& B. Wolff (Hrsg.), Körper Beratung. Beratungshandeln im Spannungsfeld von Körper, Leib und Normativität. Bielefeld: transcript.

Storch, M., Cantieni, B., Hüther, G. \& Tschacher, W. (2017). Embodiment. Die Wechselwirkung von Körper und Psyche verstehen und nutzen (3. Aufl.). Bern: Huber.

Todres, L. (2007). Embodied enquiry: phenomenological touchstones for research, psychotherapy and spirituality. Hampshire, New York: Palgrave Macmillan.

Tschacher, W. \& Storch, M. (2012). Die Bedeutung von Embodiment für Psychologie und Psychotherapie. Psychotherapie in Psychiatrie, Psychotherapeutischer Medizin und Klinischer Psychologie, 17, 17-31.

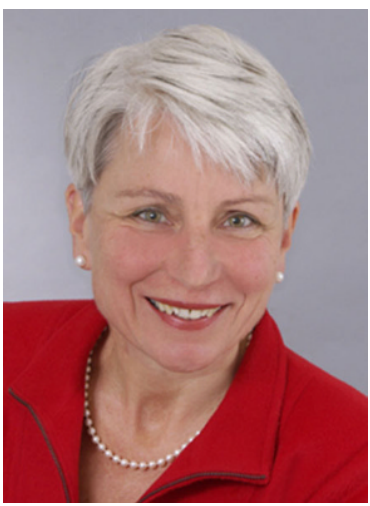

Prof. Dr. Renate Schwarz Dipl.-Sozialarbeiterin, Erziehungswissenschaftlerin, Supervisorin und Coach (DGSv), Tanztherapeutin (HPG), Yogalehrerin Yoga Vidya. Professorin für Beratung und Klinische Sozialarbeit an der RWU Hochschule Ravensburg-Weingarten, zuvor Leitung des Masterstudiengangs Psychosoziale Beratung, SRH Hochschule Heidelberg. Praxis als Sozialarbeiterin in der Suchtkrankenhilfe und im Bereich Psychiatrie. 